\title{
Surgical management of intertumor abscesses caused by fistulous communications with the intestine: rare complication in pediatric malignant tumor: 2 case reports
}

\author{
Naonori Kawakubo, MD, PhD ${ }^{a, b, \star}$, Tomoro Hishiki, MD, PhDª, Nami Shirakawa, MD ${ }^{c}$, Ayumu Arakawa, MD ${ }^{c}$, \\ Shunsuke Sugawara, MD, PhD ${ }^{d}$, Miyuki Sone, MD, PhD ${ }^{d}$, Yasuaki Arai, MD, PhD ${ }^{d}$, Chitose Ogawa, MD, $\mathrm{PhD}^{c}$, \\ Kazuaki Shimada, MD, $\mathrm{PhD}^{\mathrm{a}}$
}

\begin{abstract}
Rationale: Unresectable abdominal tumors cause various complications, and fistulizaton with intestine is a rare but serious complication. We report this condition in 2 cases.

Patient Concerns: The first case was that of a 14-year-old male patient who had a desmoid tumor and the second case was that of a 17-year-old female patient with a recurrent malignant peripheral nerve sheath tumor. They were admitted with a giant chemoresistant tumor that was gradually growing and causing abdominal pain.

Diagnoses: They were diagnosed with intertumor abscesses caused by fistulous communications with the intestine. Interventions: Both the patients underwent percutaneous drainage, and after being stabilized, they underwent surgery. Outcomes: The patient with desmoid tumor was continuing his daily routine without any problem. The patient with malignant peripheral nerve sheath tumor was able to consume food orally and discharged after the surgery.

Lessons: In the treatment of abdominal intratumor abscesses caused by fistulous communications with intestine, it is necessary to consider the optimal treatment strategies based on the tumor location and patient prognosis.
\end{abstract}

Keywords: Tumor-intestinal fistula, Desmoid tumor, Malignant peripheral nerve sheath tumor

Unresectable abdominal tumors could cause various complications as they enlarge. Bowel obstruction, bleeding, hydronephrosis, and jaundice due to compression of the biliary tract have been mainly reported $^{[1-3]}$. Fistulizaton with the intestinal tract is a rare but serious complication in such tumors. In adults, fistulization caused by gastrointestinal stromal tumor or desmoid tumor are often reported $^{[4,5]}$. However, this phenomenon is rarely reported in pediatric cases. Here, we report 2 such rare pediatric cases.

a Department of Pediatric Surgical Oncology, National Cancer Center Hospital, Tokyo, 'b Department of Pediatric Surgery, Reproductive and Developmental Medicine, Faculty of Medical Sciences, Kyushu University, Fukuoka, ' Department of Pediatric Oncology and ${ }^{d}$ Department of Diagnostic Radiology, National Cancer Center Hospital, Tokyo, Japan

Informed consent was obtained from the patients for publication of this case report and accompanying images.

This manuscript has been peer reviewed.

Sponsorships or competing interests that may be relevant to content are disclosed at the end of this article.

*Corresponding author. Address: Department of Pediatric Surgery, Reproductive and Developmental Medicine, Faculty of Medical Sciences, Kyushu University, 3-1-1, Maidashi, Higashi-ku, Fukuoka 812-8582, Japan. Tel: +81-92-642-5573. E-mail address: naonori@med.kyushu-u.ac.jp (N. Kawakubo).

Copyright @ 2020 The Authors. Published by Wolters Kluwer Health, Inc. on behalf of IJS Publishing Group Ltd. This is an open access article distributed under the terms of the Creative Commons Attribution-NonCommercial-ShareAlike 4.0 License, which allows others to remix, tweak, and build upon the work non-commercially, as long as the author is credited and the new creations are licensed under the identical terms.

International Journal of Surgery Oncology (2020) 5:e98

Received 15 July 2020; Accepted 26 July 2020

Published online 20 January 2021

http://dx.doi.org/10.1097//J9.0000000000000098

\section{Case 1}

A 14-year-old male patient with an abdominal desmoid tumor associated with Gardner syndrome was admitted with a giant chemo-resistant tumor that was gradually growing and causing abdominal pain.

He had previously undergone partial resection of the desmoid tumor located in the retroperitoneum for the treatment of ureteral rapture caused by tumor invasion. After this procedure, he had been treated with tamoxifen and non-steroidal anti-inflammatory drugs. However, the tumor enlarged, and he had been treated with $800 \mathrm{mg} /$ $\mathrm{d}$ of pazopanib in our hospital. Treatment with pazopanib achieved partial response temporarily; however, the tumor gradually enlarged after 4 months. Thus, he was administered 8 courses of methotrexate $\left(30 \mathrm{mg} / \mathrm{m}^{2}\right.$ per week) and vinblastine $\left(50 \mathrm{mg} / \mathrm{m}^{2}\right.$ per week). Despite the treatment, the tumor showed progressive disease; therefore, the surgeons considered debulking surgery. The tumor was located in the mesentery and involved the root of the superior mesenteric artery, and radical resection was considered impossible. When he was admitted to our hospital, palpation revealed tenderness in the entire abdomen. The white blood cell count was $1800 / \mu \mathrm{L}$, and C-reaction protein (CRP) level was $1.52 \mathrm{mg} / \mathrm{dL}$. Computed tomography $(\mathrm{CT})$ revealed a huge mesenteric tumor and intratumor abscess, and the abscess was connected to the intestine (Fig. 1A). Intertumor abscesses caused by fistulous communications with the intestine were suspected, and the abscess was subsequently drained percutaneously by a radiologist. A contrast study of the abscess revealed a fistula connecting to the small bowel (Fig. 1B). The patient underwent partial tumor resection and intestinal anastomosis 50 days after the drainage (Fig. 1C). However, after the surgery, leakage from the anastomosis occurred and was relieved by 

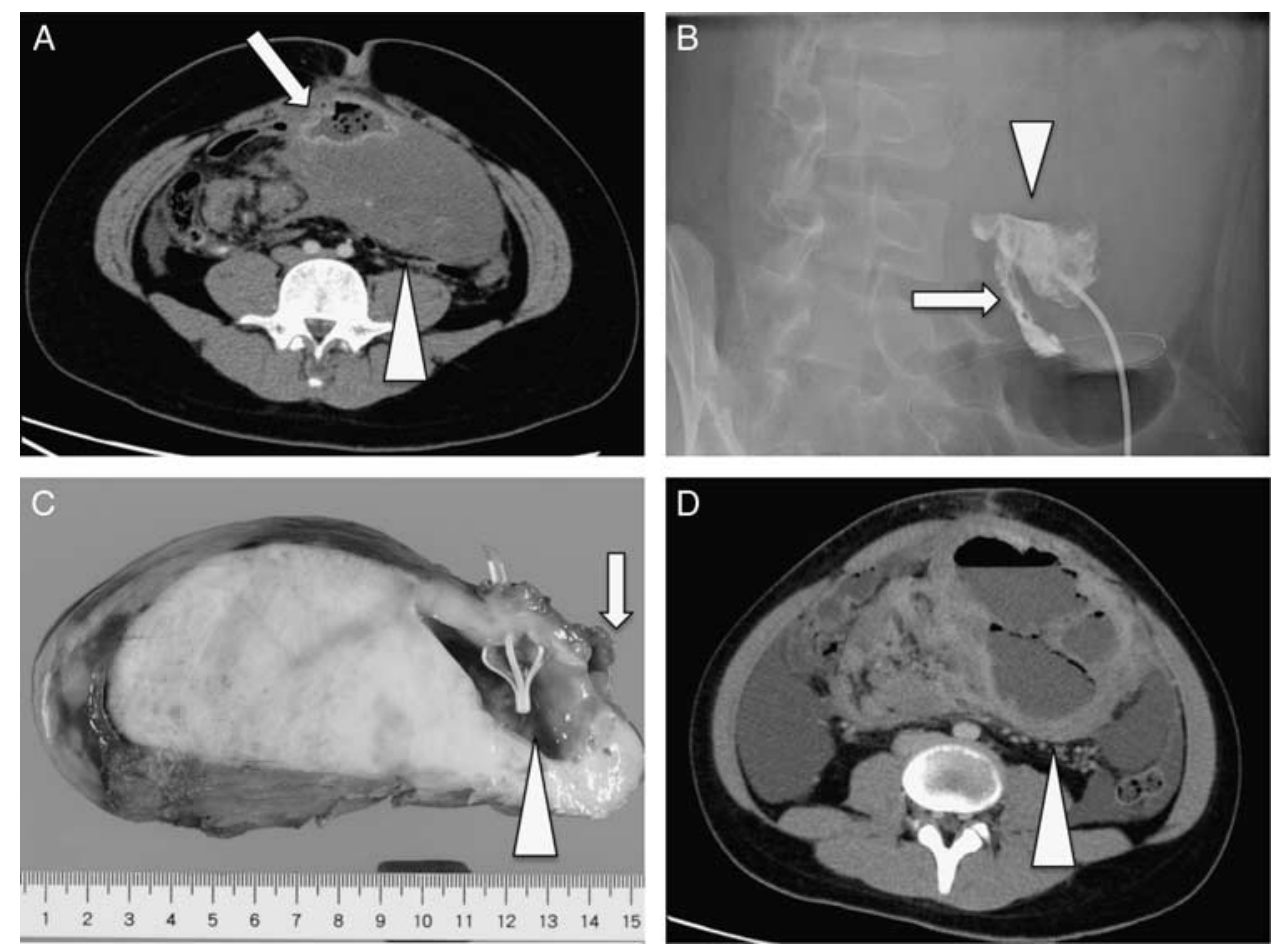

Figure 1. Computed tomography (CT) examination of case 1. A, The CT image shows intra-abdominal tumor (arrowhead) and an intratumoral abscess (arrow). $\mathrm{B}$, The contrast study from the drainage tube shows the abscess (arrowhead) and the fistulous connection into the small bowel (arrow). C, Resected tumor and intestine. The cross-section of the tumor shows the intratumoral abscess (arrowhead) and the fistula (arrow). D, The formation of an intratumor abscess (arrowhead) for the second time.

percutaneous drainage; he was discharged 90 days after admission. During the outpatient follow-up, the tumor gradually enlarged, and he underwent tumor debulking surgery 7 months after the discharge. After debulking surgery, the administration of an investigational new drug and doxorubicin $\left(37.5 \mathrm{mg} / \mathrm{m}^{2}\right)$ was started by the pediatric oncologist. However, after 1 course of medication, highgrade fever continued, and CT revealed the formation of an intratumor abscess for the second time (Fig. 1D). He underwent percutaneous drainage, and the contrast study of the abscess showed the fistula connected to the small intestine. He underwent jejunocolic anastomosis 2 weeks after the drainage. After the surgery, the abscess shrank, and we could remove the drainage tube. The pediatric oncologist continued 4 courses of doxorubicin $(37.5 \mathrm{mg} /$ $\mathrm{m}^{2}$ ), and the tumor gradually shrank but remained in the mesentery. At the time of writing this report, the patient was not receiving any treatment and was continuing his daily routine without any problem. The clinicians continue to monitor the tumor progress carefully with regular CT examinations.

\section{Case 2}

A 17-year-old female with a recurrent abdominal malignant peripheral nerve sheath tumor (MPNST) was admitted with the complaint of abdominal pain. She had previously undergone tumor resection 3 times and had received multiple courses of chemotherapy and radiotherapy. Nevertheless, the tumor had been rapidly increasing in size. The pediatric oncologist treated her with $800 \mathrm{mg} /$ $\mathrm{d}$ of pazopanib; however, the tumor gradually enlarged. Fifty days after starting pazopanib, she was admitted to our hospital. Palpation revealed tenderness in the entire abdomen. Her white blood cell count and platelet count was $12,300 / \mu \mathrm{L}$ and $7000 / \mu \mathrm{L}$, respectively. The CRP level was $17.51 \mathrm{mg} / \mathrm{dL}$. CT revealed abscess formation within the tumor (Fig. 2A) and a fistulous connection between the sigmoid colon and the tumor (Fig. 2B). She was critically ill; therefore, initially, percutaneous drainage was performed by the radiologist. Thereafter, the tumor shrank, and her condition improved. She then underwent colostomy 30 days after the percutaneous drainage. She was able to consume food orally and discharged 3 weeks after the surgery. Unfortunately, she died because of tumor enlargement 101 days after surgery.

\section{Discussion}

Complications caused by the enlargement of abdominal tumor include bleeding, bowel obstruction, hydronephrosis/hydroureter, and obstructive jaundice ${ }^{[1-3]}$. Fistulization with the intestine is rare, and few reports have been published on this subject. In particular, to our knowledge, no cases of intestinal fistulas in pediatric malignant solid tumors have been reported. Conventionally, such fistulas occured due to disease progression and as a complication of chemotherapy and radiotherapy. Recently, the advent of molecular target therapy has increased the incidence of gastrointestinal complications in malignant tumor treatment ${ }^{[4,5]}$.

In cases of desmoid tumor, several clinicians have reported fistulization ${ }^{[6-12]}$. Many patients initially underwent percutaneous drainage followed by surgery (Table 1). Bhandari and colleagues reported that if the patient condition is relatively good, percutaneous drainage is initially considered. In our case of 

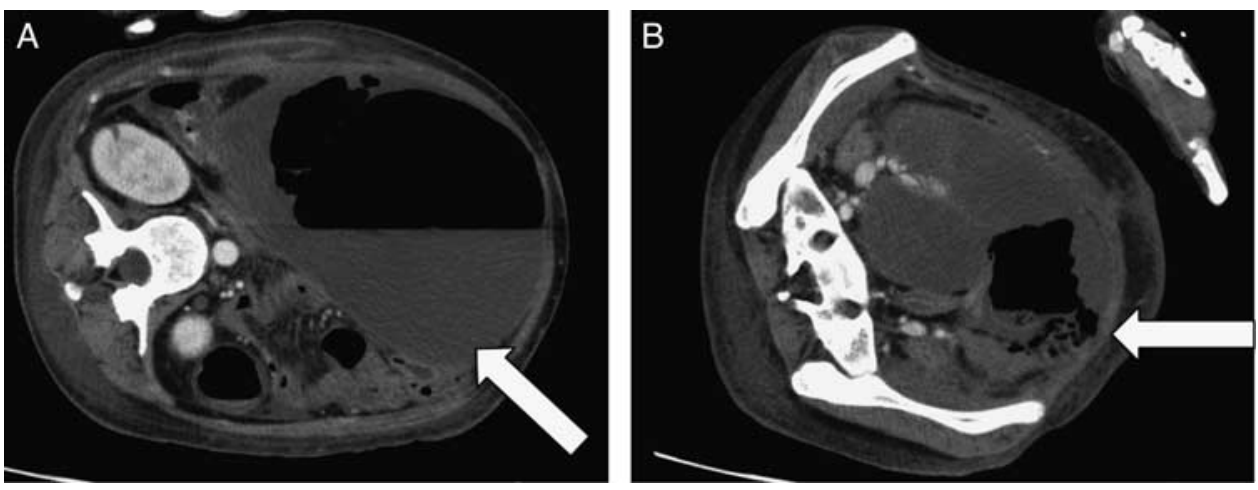

Figure 2. Computed tomography (CT) examination of case 2. A, The CT image reveals the huge tumor and intratumoral abscess with air-fluid level (arrow). $\mathrm{B}, \mathrm{A}$ fistulous connection between the sigmoid colon and the tumor (arrow).

desmoid tumor, emergency surgery was considered; however, we initially selected percutaneous drainage because the general condition of the patient was relatively good, and stoma construction could be difficult because of the giant mesenteric tumor. After percutaneous drainage, the patient's general condition improved, and a contrast study from the drainage tube enabled clear recognition of the positional relationship between the tumor and the intestinal tract before the surgery. In this case, the desmoid tumors could not be radically removed; therefore, partial tumor resection and intestinal anastomosis was initially selected. For the second fistulization, we performed anastomosis of the jejunum and the colon because approach to the fistula and the surrounding tumor was very challenging, and we believed that minimally invasive surgery should be performed to resume chemotherapy immediately.

To our knowledge, there has been no report on tumor-intestinal fistula in case of MPNST. In our case, fistulization might have resulted from tumor compression/invasion and/or as the adverse

\section{Table 1}

The cases of tumor-intestinal fistula in desmoid tumor.

\begin{tabular}{|c|c|c|c|c|}
\hline References & $\begin{array}{l}\text { No. } \\
\text { cases }\end{array}$ & $\begin{array}{l}\text { Age } \\
\text { (y) }\end{array}$ & $\begin{array}{c}\text { FAP } \\
\text { associated }\end{array}$ & Procedure \\
\hline \multirow[t]{3}{*}{ Maldjian et al ${ }^{[6]}$} & 3 & NA & Y & Drainage $\rightarrow$ surgery \\
\hline & & NA & Y & Drainage $\rightarrow$ surgery \\
\hline & & NA & Y & Drainage \\
\hline Cholongitas et al ${ }^{[7]}$ & 1 & 32 & N & Drainage $\rightarrow$ surgery \\
\hline Ebrahimi-Daryani et al ${ }^{[8]}$ & 1 & 54 & N & Antibiotics $\rightarrow$ surgery \\
\hline \multirow[t]{9}{*}{ Bhabdari et al ${ }^{[9]}$} & 9 & 37 & Y & Drainage $\rightarrow$ surgery \\
\hline & & 22 & Y & Drainage \\
\hline & & 24 & Y & Surgery \\
\hline & & 31 & Y & Drainage $\rightarrow$ surgery \\
\hline & & 24 & Y & Drainage \\
\hline & & 41 & Y & Nonoperative \\
\hline & & 24 & Y & Surgery \\
\hline & & 20 & Y & Surgery \\
\hline & & 36 & Y & Nonoperative \\
\hline Peled et $\mathrm{al}^{[10]}$ & 1 & 32 & N & Antibiotics $\rightarrow$ surgery \\
\hline Alemanno et al ${ }^{[11]}$ & 1 & 38 & Y & Drainage \\
\hline Huang et $\mathrm{al}^{[12]}$ & 1 & 36 & N & Drainage $\rightarrow$ surgery \\
\hline \multirow{2}{*}{ Our case } & 2 & 14 & Y & Drainage $\rightarrow$ surgery \\
\hline & & 16 & Y & Drainage $\rightarrow$ surgery \\
\hline
\end{tabular}

FAP indicates familial adenomatous polyposis; $\mathrm{N}, \mathrm{no} ; \mathrm{NA}$, not available; $\mathrm{Y}$, yes. effect of pazopanib: multi kinase inhibitor. Several molecular target agents, including pazopanib have antiangiogenic properties that influence their efficacy as well as complications. Bowel-related complications, such as colitis, enteritis, pneumatosis, bowel perforation, and fistulization are drug-specific toxicities of antiangiogenic agents, such as bevacizumab, sunitinib, sorafenib, and pazopanib $b^{[4,5,13]}$.

For the patient with MPNST, drainage was initially selected because the patient's general condition was poor. Drainage improved the general condition and reduced the size of the intraabdominal tumor, enabling stoma construction. Radical resection was difficult because of the huge tumor, and poor prognosis. Thus, we performed stoma construction, not anastomosis of the colon.

Radical resection of the tumor and fistulized intestine is often impossible because of the tumor location, size, and spread. In such cases, presurgical interventions, such as percutaneous drainage, are necessary. The contrast study from the drainage tube is useful for clarifying the positional relationship of the tumor and the intestine. Surgeons need to carefully consider the surgical procedure with respect to the findings of the $\mathrm{CT}$ and the contrast study.

When treating abdominal intratumor abscesses caused by fistulous communications with the intestine, it is necessary to consider the optimal treatment strategies based on the tumor location, the anatomic relationship of the tumor and the fistula, and patient prognosis.

\section{Ethical approval}

Informed consent was obtained from the patients for publication of this case report and accompanying images.

\section{Sources of funding}

None.

\section{Author contribution}

N.K.: conceptualization, investigation, methodology, and writingoriginal draft. T.H. and K.S.: project administration. N.S., A.A., C.O.: resources. K.S.: supervision. S.S., M.S., Y.A.: visualization. T.H.: writing — review and editing. 


\section{Conflict of interest disclosure}

The authors declare that they have no financial conflict of interest with regard to the content of this report.

\section{Research registration unique identifying number (UIN)}

researchregistry 6427.

\section{Guarantor}

None.

\section{References}

[1] Handa A, Nozaki T, Makidono A, et al. Pediatric onocologic emergencies: clinical and imaging review for pediatricians. Pediatr Int 2019;61: 122-39.

[2] Katabethina VS, Restrepo CS, Betancourt Cuellar SL, et al. Imaging of oncologic emergencies: what every radiologist should know. Radiographics 2013;33:1533-53.

[3] Szavay P, Kurth R, Schaefer J, et al. A rare cause for extrahepatic biliary tract obstruction: Juvenile hepatic hilar hemangioma. Eur J Pediatr Surg 2013;23:415-7.
[4] Tirumani SH, Baez JC, Jagannathan JP, et al. Tumor-bowel fistula: what radiologists should know. Abdom Imaging 2103;38:1014-23.

[5] Tirumani SH, Shinagare AB, Jagannathan JP, et al. Multidetector-row CT of tumor-bowel fistula: experience at a teritiary cancer centre. Clin Radiol 2014;69:e100-7.

[6] Maldjian C, Mitty H, Garten A, et al. Abscess formation in desmoid tumors of Gardner's syndrome and percutaneous drainage: a report of three cases. Cardiovasc Intervent Radiol 1995;18:18-171.

[7] Cholongitas E, Koulenti D, Panetsos G, et al. Desmoid tumor presenting as intra-abdominal abscess. Dig Dis Sci 2006;51:68-9.

[8] Ebrahimi-Daryani N, Momeni A, AziziAsl MR, et al. Mesenteric fibromatosis (desmoid tumor) presenting as recurrent abdominal abscess: report of a rare case. Govaresch 2008;13:128-32.

[9] Bhandari S, Ranchod P, Sinha A, et al. Familial adenomatous polyposisrelated desmoids presenting with air-fluid level: a clinical review and management algorithm. Dis Colon Rectum 2012;55:810-4.

[10] Peled Z, Linder R, Gilshtein H, et al. Cecal fibomatosis(desmoid tumor) mimicking periappendicular abscess: a case report. Case Rep Oncol 2012;5:511-4.

[11] Alemanno G, Zambonin D, Sturiale A, et al. A multidisciplinary approach to desmoid tumors when intra-abdominal fibromatosis degenerates into an abscess, which is the right treatment? Int J Surg Case Rep 2013;4:757-60.

[12] Huang K, Stuart H, Lyapichev K, et al. Mesenteric desmoid tumour presenting with recurrent abdominal abscess and duodenal fistula: a case report and review of literature. Int J Surg Case Rep 2017;37:119-23.

[13] Pereira ROL, Patel PR, Guru PK, et al. Tumour-bowel fistula as a possible complication of pazopanib therapy in retroperitoneal leiomyosarcoma. BMJ Case Rep 2019;12:pii: e230727. 